\title{
Research on the Synchronization Control Strategy for Microgrid-Connected Voltage Source Inverter
}

\author{
Yuanzhi Cao \\ Department of Renewable Energy \\ China Electric Power Research \\ Institute \\ Nanjing, China \\ caoyuanzhi@epri.sgcc.com.cn
}

\author{
Yanting $\mathrm{Hu}$ \\ Faculty of Science and Engineering \\ University of Chester \\ Chester, UK \\ y.hu@chester.ac.uk
}

\author{
Rui Hu, Jianfei Chen \\ Department of Energy Engineering \\ Aalborg University \\ Aalborg, Denmark \\ rhu@et.aau.dk,jia@et.aau.dk
}

\begin{abstract}
Microgrid is intended and featured to be able to operate in both grid-connected and islanded mode to ensure high quality and reliable power supply. In order to achieve stable operation of the microgrid-connected voltage source inverter (MVSI) units under paralleled or grid-connected mode, a novel synchronization method based on droop control is proposed in this paper. The difference of phase and amplitude between different MVSI units is detected and is used to calculate the output frequency and amplitude of the MVSI. This method can smooth transfer the MVSI units from standalone mode to paralleled mode. The simulation and experimental results show that the proposed method is effective in achieving paralleled operation of the MVSI units.
\end{abstract}

Keywords-microgrid; grid-connected mode; islanded mode; voltage source inverter; synchronization method

\section{INTRODUCTION}

With the rapid development of renewable power generation technology, such as solar and wind power generation, distributed generation (DG) has been more and more widely applied. Added by energy storage devices, DGs can provide power to a local load, thus forming a microgrid [1]. In order to provide high quality and reliable power supply, the microgrid should not only be able to operate in gridconnected mode, but also islanded mode to provide power for local loads when it is necessary[2]. Thus, most of the Microgrid-connected Voltage Source Inverters (MVSIs) are required to have the capability to enable the microgrid to operate in either mode, a) grid-tied mode to share load among them as well as subsist under transient events of the system, b) stand-alone mode to provide power during utility outages until service can be restored [3]. As a result, seamless transfer between the two operating modes is of significance [4].

MVSIs are very interesting for DG applications since they do not need any external reference to stay synchronized [5], [6]. In fact, they can operate in parallel with other inverters by using frequency and voltage droops, forming autonomous or stand-alone microgrid [7]. When these MVSIs are required to operate in grid-connected mode, they often change its behavior from voltage to current sources [8]. Nevertheless, to achieve flexible microgrid, i.e., able to operate in both gridtied and stand-alone modes, MVSIs are required to control the power exported to or imported from the utility and to stabilize the microgrid [9]. In this sense, the droop method can be used to inject active and reactive power from the MVSI to the grid by adjusting the frequency and amplitude of the output voltage [10], [11].

To ensure seamless transfer between grid-connected and islanded operation modes, the voltages of different MVSI units should be synchronized in frequency, phase and amplitude [12-15]. This paper presents a synchronization method based on droop control and some research results obtained from simulations and experimentation by applying this method into the studied system. The rest of this paper is arranged as follows. Section II introduces the study structure of microgrid and paralleled MVSI units, systematically describes the droop strategy. Section III expounds the synchronization method based on Phase Lock Loop (PLL). Section IV provides simulation and experimental results from two 20kVA MVSIs parallel system. Section V concludes this paper.

\section{THE SYSTEM STRUCTURE OF MVSI UNITS BASED ON DROOP CONTROL}

\section{A. Structure and Analysis of Microgrid}

The studied microgrid structure is shown in Fig. 1, which consists of two DGs and AC loads connected to the utility grid. Power electronic interface unit (DC/AC or AC/DC/AC and the control/drive) is connected in between each $\mathrm{DG}$ and the point of common coupling (PCC), considering the necessities of most DGs in microgrid. The states of $\mathrm{CB}$, open or close, decide the microgrid's operation mode.

In islanded mode, the control switch is open. While in grid-connected mode, the control switch is closed.

\section{B. The Parallel System Structure of MVSI Units}

The equivalent parallel structure of MVSI units is shown in Fig. 2. It includes the two MVSI units and the AC $\operatorname{load} Z_{\text {load }}$. Each MVSI unit contains a filter inductance $L$, a filter capacitor $C$ and the equivalent DC source $U_{d c}$ at the inverter's DC side. 


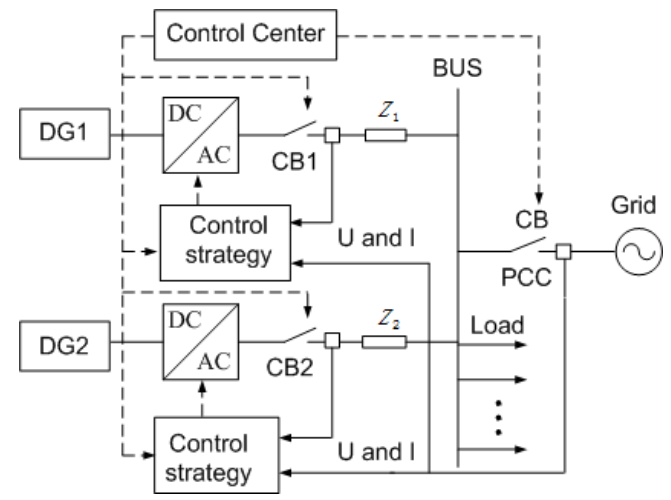

Fig. 1. Microgrid structure.

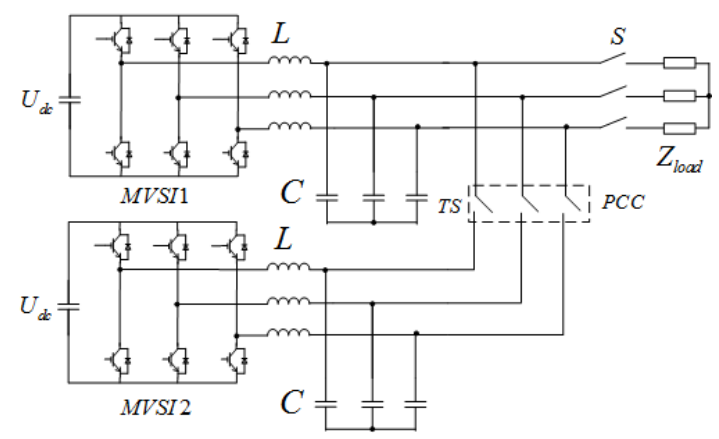

Fig. 2. The parallel structure of two MVSI units.

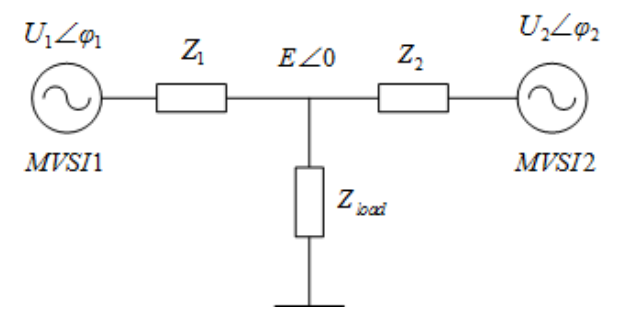

Fig. 3. Equivalent circuit of two MVSI units parallel operation.

\section{The Conventional Droop Control Scheme}

To simplify the analysis, the configuration of two MVSI units sharing a common load $Z_{\text {load }}$ is taken as an example, and the equivalent circuit is shown in Fig.3. $U_{1} \angle \varphi_{1}$ and $U_{2} \angle \varphi_{2}$ are MVSI1 and MVSI2 output voltages, $Z_{1}$, $Z_{2}$ represent inverter control output impedance, $E \angle 0$ is the microgrid voltage.

The output active power and reactive power of each MVSI can be calculated according to the equivalent circuit established as:

$$
\left\{\begin{array}{l}
P=\frac{E U}{Z} \cos (\phi-\varphi)+\frac{E^{2}}{Z} \cos \phi \\
Q=\frac{E U}{Z} \sin (\phi-\varphi)-\frac{E^{2}}{Z} \sin \phi
\end{array}\right.
$$

Assuming the output impedance $Z_{1}$ and $Z_{2}$ are pure reactive $X$, that is the output impedance phase $\phi=90^{\circ}$, then the active and reactive power flowing out of every MVSI module can be expressed as:

$$
\left\{\begin{array}{c}
P=\frac{E U}{X} \sin \varphi \\
Q=\frac{E U \cos \varphi-E^{2}}{X}
\end{array}\right.
$$

Equation (2) indicates that if the phase difference $\varphi$ between the MVSI output voltage vector $U$ and the microgrid voltage vector $\mathrm{E}$ is very small, it can be approximated as $\sin \varphi \approx \varphi, \cos \varphi \approx 1$, then apply to (2), the active power and reactive power can be simplified as:

$$
\left\{\begin{array}{c}
P=\frac{E U}{X} \sin \varphi \approx \frac{E U}{X} \varphi \\
Q=\frac{E U \cos \varphi-E^{2}}{X} \approx \frac{E U-E^{2}}{X}
\end{array}\right.
$$

The pair of equation in (3) indicate that the active power flow $P$ is largely dependent on the phase difference while the reactive power flow $Q$ is largely dependent on the amplitude of the inverter voltage $U$. That is the theory basis of droop method, which can be expressed as equation (4).

$$
\left\{\begin{array}{l}
\omega=\omega_{0}+m\left(P_{r e f}-P\right) \\
U=U_{0}+n\left(Q_{r e f}-Q\right)
\end{array}\right.
$$

Where $\omega_{0}$ and $U_{0}$ are the reference value of the inverter frequency and voltage, $P_{r e f}$ and $Q_{\text {ref }}$ are the reference value of the output active and reactive power, $m$ and $n$ are the active and reactive droop coefficients. The relationship between the frequency $\omega$ and position $\theta$ is

$$
\frac{d \theta}{d t}=\omega
$$

Angle $\theta$ is required for the droop modulation scheme. The droop control approach, based on equations (1) to (5), is represented by the block diagram of Fig. 4 .

\section{A NEW SYNCHRONIZATION METHOD}

A new synchronization method based on PLL for paralleled MVSI units is presented in Fig. 5, where the microgrid voltage vector $e_{a}, e_{b}, e_{c}$ is transformed from the $\alpha \beta$ stationary reference frame to the $d q$ rotating reference frame by means of Park's transformation, that is

$$
\begin{aligned}
& {\left[\begin{array}{c}
E_{d} \\
E_{q}
\end{array}\right]=\left[\begin{array}{cc}
\cos \theta & \sin \theta \\
-\sin \theta & \cos \theta
\end{array}\right]\left[\begin{array}{c}
e_{\alpha} \\
e_{\beta}
\end{array}\right]=} \\
& {\left[\begin{array}{cc}
\cos \theta & \sin \theta \\
-\sin \theta & \cos \theta
\end{array}\right]\left[\begin{array}{c}
E \cos \theta_{e} \\
E \sin \theta_{e}
\end{array}\right]=\left[\begin{array}{c}
E \cos \left(\theta-\theta_{e}\right) \\
E \sin \left(\theta-\theta_{e}\right)
\end{array}\right]}
\end{aligned}
$$




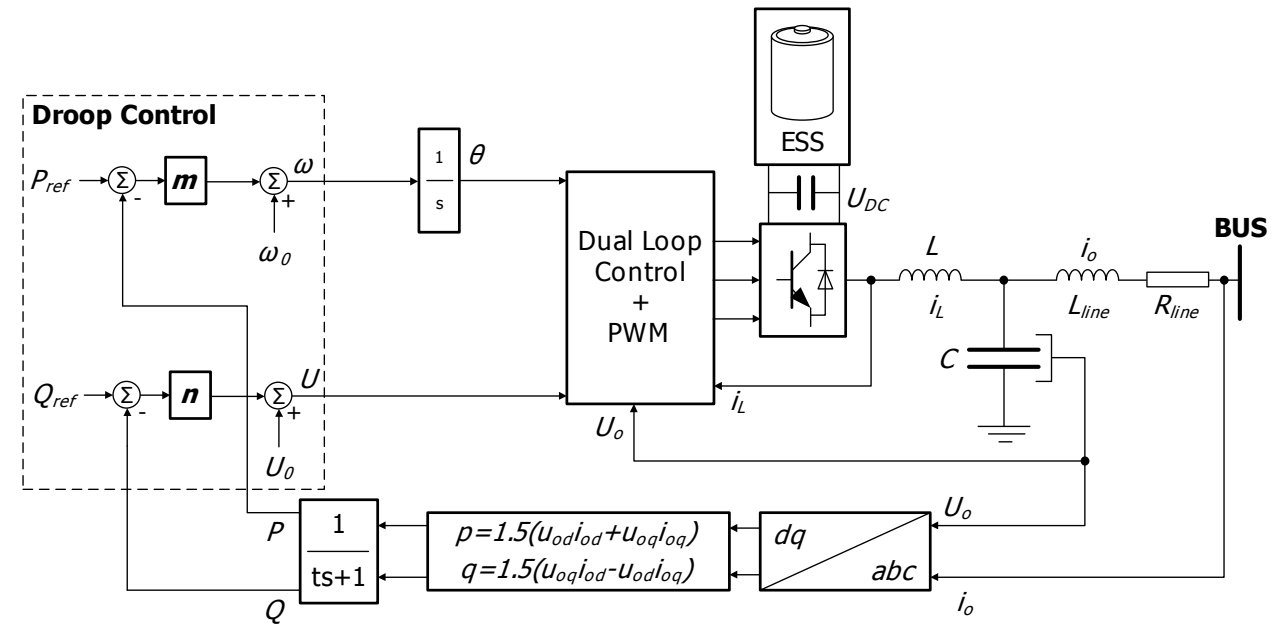

Fig. 4. Block diagram of the MVSI unit based on Droop Control.

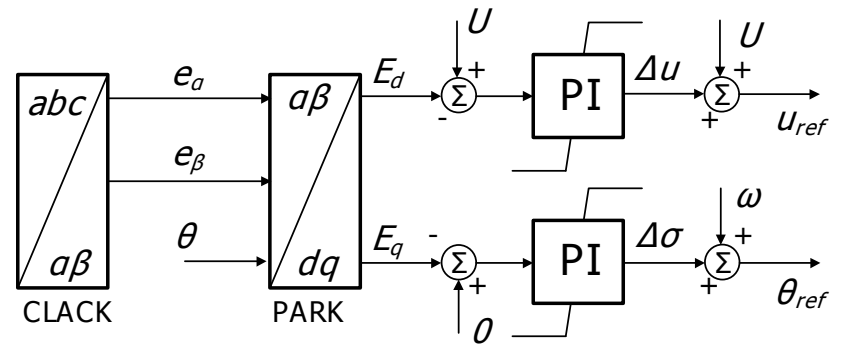

Fig. 5. Block diagram of the synchronization method based on Phase Lock Loop.

The principle of generating the frequency offset $\Delta \sigma$ and amplitude offset $\Delta u$ is as follows according to (6). The $q$ axis component $E_{q}$ of voltage vector $E$ is used to do subtraction with 0 , then the result is put into PI regulator to get $\Delta \sigma$, while the $d$ axis component $E_{d}$ is used to do subtraction with $U$, and then the result is also put into PI regulator to obtain $\Delta u$ is shown in Fig. 5. The frequency and amplitude of $u_{r e f}$ is respectively determined by the $\Delta \sigma$ and the $\Delta u$ as follow

$$
\left\{\begin{array}{c}
\omega_{r e f}=\omega+\Delta \sigma=\omega_{0}+m\left(P_{r e f}-P\right)+\Delta \sigma \\
u_{r e f}=U+\Delta u=U_{0}+n\left(Q_{r e f}-Q\right)+\Delta u
\end{array}\right.
$$

According to (7), at any time $T_{x}$, the amount that the difference of phase and amplitude has been reduced can be expressed as

$$
\left\{\begin{array}{l}
\Delta \omega=\int_{0}^{T x}[(\omega+\Delta \sigma)-\omega] \cdot d t=\int_{0}^{T x} \Delta \sigma \cdot d t \\
\Delta U=\int_{0}^{T x}[(U+\Delta u)-U] \cdot d t=\int_{0}^{T x} \Delta u \cdot d t
\end{array}\right.
$$

It can be seen from (8) that the shrinking of $\Delta \omega$ and $\Delta U$ is the product of the integral of last $\Delta \sigma$ and $\Delta u$ changing with time. At last, $\Delta \sigma$ and $\Delta u$ will trend to zero and realize the synchronization of phase and amplitude. The equivalent control block diagram is shown in Fig. 6.

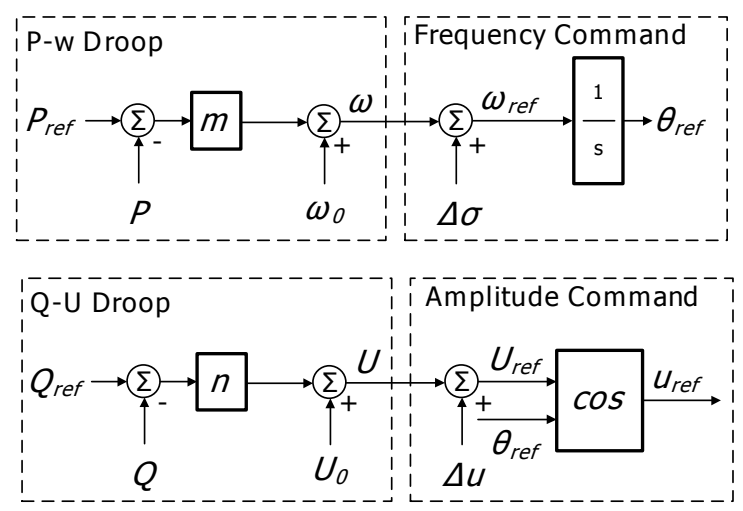

Fig. 6. The equivalent block diagram of synchronization controller.

TABLE I. KeY PARAMETERS OF THE MVSI PrototyPE

\begin{tabular}{ccc}
\hline Symbol & Meaning & Value \\
\hline$S_{n}$ & Inverter capacity & $20 \mathrm{KVA}$ \\
$C$ & Filter capacitor & $100 \mathrm{uF}$ \\
$L$ & Filter inductor & $2.5 \mathrm{mH}$ \\
$\omega_{0}$ & System frequency & $314 \mathrm{rad} / \mathrm{s}$ \\
$U_{0}$ & System voltage & $220 \mathrm{~V}$ \\
$f_{s w}$ & Switch frequency & $10 \mathrm{KHZ}$ \\
$f_{c}$ & Sample frequency & $10 \mathrm{KHZ}$ \\
\hline
\end{tabular}

IV. SimUlATION AND EXPERIMENTAL RESUlTS

To verify the strategy introduced in III a paralleled system according to Fig. 2 is built with Matlab/Simulation and MVSI1 and MVSI2 are controlled with droop method. The main parameters of the system are shown in Table I.

The MVSI1 works at $0 \mathrm{~s}$ with a $10 \mathrm{KW}$ load and the MVSI2 is connected to MVSI1 when synchronization is 
completed. Fig. 7 shows the output voltage of the two MVSI units during synchronization procedure. At about $0.15 \mathrm{~s}$, the two output voltages are synchronized in both phase and amplitude. The output currents of the two MVSI units are presented in Fig. 8. It is shown that the two MVSI units do not generate inrush current when the system stays synchronized. Fig. 9 shows the active power of the two MVSI units; it also shows that the good power-sharing capability can be achieved quickly when the synchronization process is completed.

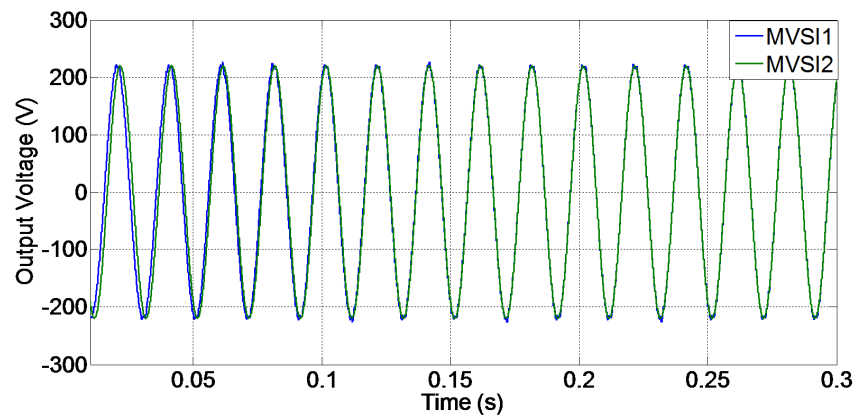

Fig. 7. Output voltages of MVSI1 and MVSI2 during the synchronization procedure.

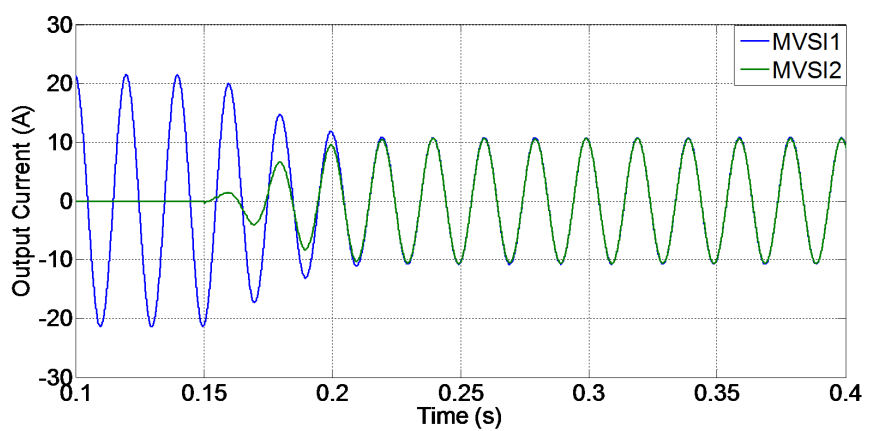

Fig. 8. Output current of MVSI1 and MVSI2.

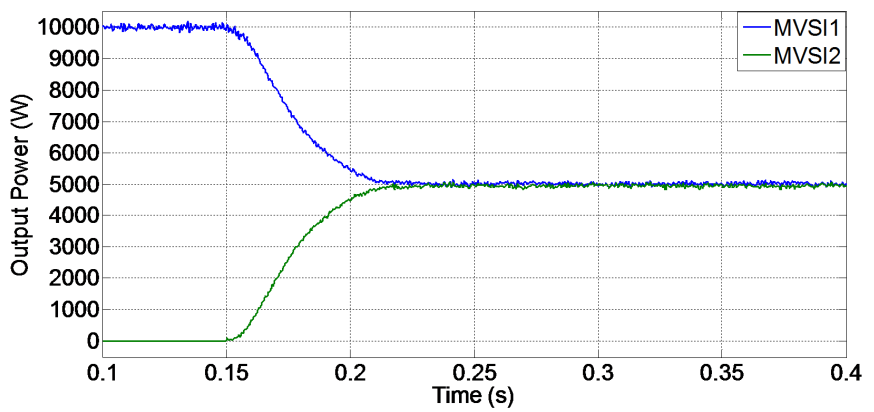

Fig. 9. Active power of MVSI1 and MVSI2.

Two 20kVA MVSI units were built and tested according to Fig. 2, with the droop control strategy applied to the synchronization controller which is based on PLL and implemented with a TMS320LF28335 DSP. The main parameters of the system are also shown in Table I. Fig.10 is the experimental result of the output voltage during synchronization procedure. Fig. 11 and Fig. 12 respectively show the measured output current and output active power of each MVSI unit. The experimental results also verify that the proposed method can achieve synchronization and good power-sharing without inrush current.

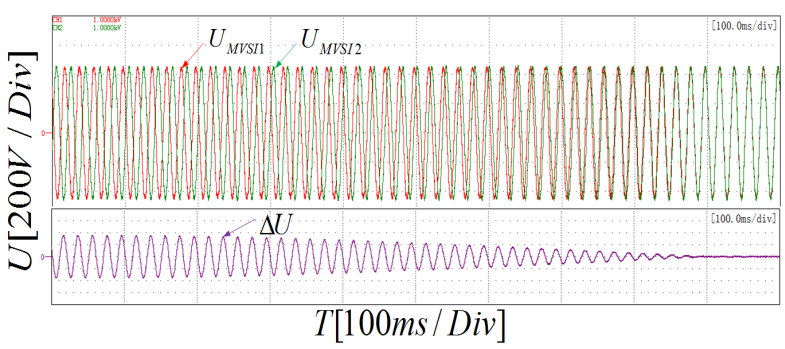

Fig. 10. Output voltage experimental result of MVSI1 and MVSI2 during the synchronization procedure.

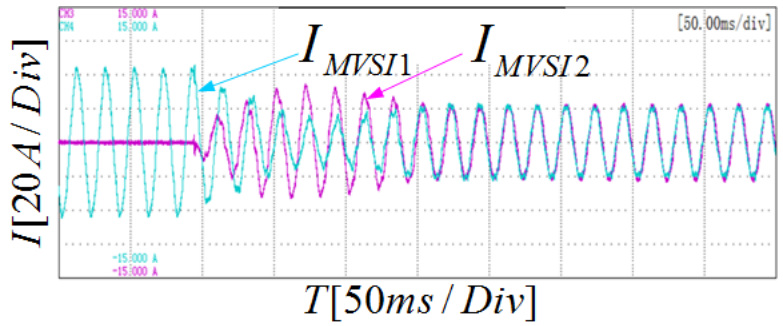

Fig. 11. Measured output current of MVSI1 and MVSI2.

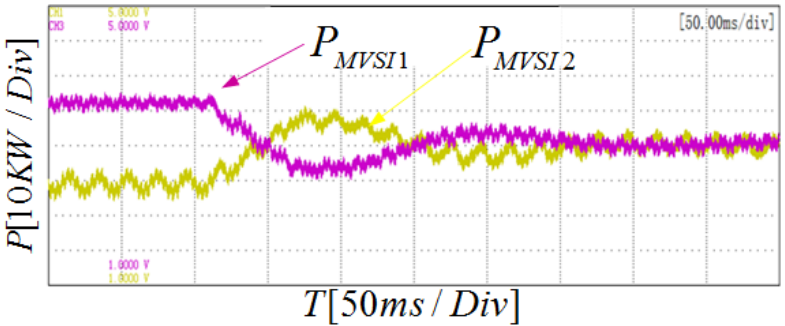

Fig. 12. Measured active power of MVSI1 and MVSI2.

\section{CONCLUSION}

A new synchronization controller based on PLL is designed in this paper to achieve the MVSI units switching from standalone mode to paralleled mode. This method not only generates no inrush current during synchronization procedure but also achieves a good power-sharing capability quickly. Simulation and experimental results are presented to confirm the effectiveness of the method.

\section{REFERENCES}

[1] Guerrero, J.M.; Vasquez, J.C.; Matas, J.; Castilla, M.; de Vicuna, L.G., "Control Strategy for Flexible Microgrid Based on Parallel LineInteractive UPS Systems,"Industrial Electronics, IEEE Transactions on vol.25,no.3, pp.726-736,March 2009.

[2] Haizhen Xu; Xing Zhang; Fang Liu; Debin Zhu; Rongliang Shi; Hua $\mathrm{Ni}$; Wei Cao, "Synchronization strategy of microgrid from islanded to grid-connected mode seamless transfer," TENCON 2013 - 2013 IEEE Region 10 Conference (31194), vol., no., pp.1,4, 22-25 Oct. 2013.

[3] Yaoqin Jia; Dingkun Liu; Jia Liu, "A novel seamless transfer method for a microgrid based on droop characteristic adjustment," Power Electronics and Motion Control Conference (IPEMC),2012 7th International, vol.1, no., pp.362,367, 2-5 June 2012. 
[4] Zhilei Yao; Lan Xiao; Yangguang Yan, "Seamless Transfer of SinglePhase Grid-Interactive Inverters Between Grid-Connected and StandAlone Modes," Power Electronics, IEEE Transactions on , vol.25, no.6, pp.1597,1603, June 2010.

[5] Mohamed, Y.A.-R.I.; El-Saadany, E.F., "A Control Scheme for PWM Voltage-Source Distributed-Generation Inverters for Fast Load-Voltage Regulation and Effective Mitigation of Unbalanced Voltage Disturbances," Industrial Electronics, IEEE Transactions on, vol.55, no.5, pp.2072,2084, May 2008.

[6] Lazzarin, T.B.; Bauer, G.A.T.; Barbi, I., "A Control Strategy for Parallel Operation of Single-Phase Voltage Source Inverters: Analysis, Design and Experimental Results," Industrial Electronics, IEEE Transactions on, vol.60, no.6, pp.2194,2204, June 2013.

[7] Luo, F.; Lai, Y.M.; Loo, K.H.; Tse, C.K.; Xinbo Ruan, "A generalized droop-control scheme for decentralized control of inverter-interfaced microgrids," Circuits and Systems (ISCAS), 2013 IEEE International Symposium on , vol., no., pp.1320,1323, 19-23 May 2013.

[8] Ochs, David S.; Sotoodeh, Pedram; Mirafzal, Behrooz, "A technique for voltage-source inverter seamless transitions between grid-connected and standalone modes," Applied Power Electronics Conference and Exposition (APEC), 2013 Twenty-Eighth Annual IEEE, vol., no., pp.952,959, 17-21 March 2013.

[9] Guerrero, J.M.; Matas, J.; Luis Garcia de Vicuna; Castilla, M.; Miret, J., "Decentralized Control for Parallel Operation of Distributed Generation Inverters Using Resistive Output Impedance," Industrial Electronics, IEEE Transactions on , vol.54, no.2, pp.994,1004, April 2007.

[10] Yaoqin Jia, Dingkun Liu, and Jia Liu, "A novel seamless transfer method for a microgrid based on droop characteristic adjustment," Power Electronics and Motion Control Conference (IPEMC), 2012 7th International, vol.1, no., pp.362-367, Jun. 2012.

[11] Cheng Jin, Mingzhi Gao, Xiaofeng Lv, and Min Chen, "A seamless transfer strategy of islanded and grid-connected mode switching for microgrid based on droop control," Energy Conversion Congress and Exposition (ECCE), 2012 IEEE, vol., no., pp.969-973, Sept. 2012.

[12] Rongliang Shi; Xing Zhang; Haizhen Xu; Fang Liu; Wei Cao, "Research on the synchronization control strategy for microgrid inverter," Electronics and Application Conference and Exposition (PEAC), 2014 International , vol., no., pp.210,213, 5-8 Nov. 2014.

[13] Mahmood, H., and Jin Jiang, "A control strategy of a distributed generation unit for seamless transfer between grid connected and islanded modes," Industrial Electronics (ISIE), 2014 IEEE 23rd International Symposium on, vol., no., pp.2518-2523, Jun. 2014.

[14] Zhiqiang Guo, Deshang Sha, and Xiaozhong Liao, "Voltage magnitude and frequency control of three-phase voltage source inverter for seamless transfer," Power Electronics, IET, vol.7, no.1, pp.200-208, Jan. 2014.

[15] Thanh-Vu Tran, Tae-Won Chun, Hong-Hee Lee, Heung-Geun Kim, and Eui-Cheol Nho, "PLL-Based Seamless Transfer Control Between GridConnected and Islanding Modes in Grid-Connected Inverters," IEEE Trans. Power Electron., vol.29, no.10, pp.5218-5228, Oct. 2013. 